\title{
PENFIGÓIDE BOLHOSO INDUZIDO POR FÁRMACO: UM RELATO DE CASO
}

\author{
Danielle F. Lonchiati, Beatriz S. de Oliveira, Andressa L. F. Silva, Beatrice S. Souza, Murilo O. Carapeba, Luíza \\ R. Pivaro, Leandra E. Kerche
}

Universidade do Oeste Paulista, Faculdade de Medicina, Presidente Prudente-SP, Brasil. e-mail: leakerche@unoeste.br

\section{RESUMO}

O penfigóide bolhoso é a mais comum dermatose autoimune podendo ser desencadeada por fármacos. 0 objetivo desse estudo foi acompanhar a evolução de uma paciente com farmacodermia em uso de diversos medicamentos. Paciente do sexo feminino foi atendida em um hospital na cidade de Presidente Prudente, apresentando bolhas íntegras e rotas, necessitando de investigação etiológica. Foram descartadas outras hipóteses diagnósticas como porfiria cutânea tarda e através da biópsia cutânea de uma bolha foi sugerido o diagnóstico de penfigóide bolhoso de etiologia farmacológica. Suspendemos o uso de antibióticos e mantivemos a medicação de uso contínuo. Foi introduzida prednisona oral e dexametasona tópico, podendo observar evolução favorável do quadro, sem intercorrências, com diminuição das bolhas até restar apenas lesões residuais. A análise das variáveis do caso permitiu pressupor que as quinolonas foram as responsáveis pelo quadro, ressaltando a importância de conhecer as reações medicamentosas para garantir um diagnóstico precoce.

Palavras-chave: Doenças de pele, penfigóide bolhoso, erupção medicamentosa, dermatose bolhosa, quinolonas.

\section{DRUG-INDUCED BULLOUS PEMPHIGOID: A CASE REPORT}

\begin{abstract}
Bullous pemphigoid is the most common autoimmune dermatosis that can be caused by drugs. The objective of this study was following the evolution of a patient with pharmacodermia in use of several drugs. A female patient was treated in a hospital in the city of Presidente Prudente, presenting intact and broken blisters, require etiological investigation. Other diagnostic hypotheses such as porphyria cutanea tarda were ruled out, and the diagnosis of bullous pemphigoid of pharmacological etiology was suggested through cutaneous biopsy of a blister. We stopped using antibiotics and kept the medication in continuous use. It was introduced oral prednisone and topical dexamethasone, and it was possible to observe a favorable evolution of the condition, without intercurrences and reduction of the blisters until only residual lesions remained. By analyzing the variables of the case it is assumed that the quinolones were responsible for the condition, emphasizing the importance of knowing the drug reactions to guarantee an early diagnosis.
\end{abstract}

Keywords: Skin diseases, pemphigoid bullous, drug eruptions, linear IgA bullous dermatoses, quinolones.

\section{INTRODUÇÃO}

As farmacodermias são doenças tegumentares (pele e/ou mucosas) e/ou sistêmicas, produzidas direta ou indiretamente pelo uso de medicamentos introduzidos no organismo por ingestão, injeção, inalação, instilação ou contato ${ }^{1}$. São conhecidas como reações de hipersensibilidade, as quais se enquadram na categoria de reações adversas a drogas. Essas reações de hipersensibilidade são inerentes à resposta imunológica do indivíduo e são classificadas em tipo I, II, III, IV e não 
classificada. As reações de hipersensibilidade do tipo I são desencadeadas pela ligação da droga ou do hapteno a duas moléculas de IgE específica, que, por sua vez, ligam-se aos mastócitos, causando sua degranulação e ativação, liberando histamina e outros mediadores inflamatórios, que são responsáveis pelas manifestações clínicas. Normalmente, as lesões se manifestam por pápulas eritematoedematosas e pruriginosas ${ }^{2}$.

As reações a drogas são, geralmente, associadas a lesões cutâneas, porque a pele é um órgão imunológico metabolicamente ativo, podendo então apresentar angioedema, urticária, eczema, eritema nodoso, exantema morbiliforme e penfigóide bolhoso. A história e o exame físico são as ferramentas mais importantes para se chegar ao diagnóstico de hipersensibilidade à droga, portanto deve ser feita uma relação completa de todos os medicamentos tomados pelo paciente na ocasião e as datas da administração; quando há várias drogas envolvidas, deve-se optar como causa mais provável a droga mais comumente relacionada à hipersensibilidade, e pela droga introduzida por último. O tratamento consiste na retirada da droga envolvida, e o uso de corticosteróides ou de anti-histamínicos deverá ser indicado de acordo com o tipo de hipersensibilidade envolvida ${ }^{2}$.

O penfigóide bolhoso é a dermatose bolhosa auto-imune mais comum ${ }^{3-6}$, caracterizada por uma resposta auto-imune em relação a duas proteínas presentes nos hemidesmossomos na zona da membrana basal, que são BP180 e $\mathrm{BP} 230^{7}$, levando a acantólise, que é a perda de adesão célula-célula, resultando em separação intra-epidérmica ${ }^{8}$. Fatores genéticos e ambientais contribuem para a susceptibilidade à doença, e sua patogênese tem sido associada a muitos fatores exógenos, como a ingestão de drogas e infecções virais ${ }^{5}$, no entanto, a validação desses fatores na patogênese do penfigóide bolhoso não está totalmente estabelecida ${ }^{6}$.

A doença afeta principalmente pessoas com mais de 70 anos de idade ${ }^{4-6,8}$, sem predileção étnica, racial ou sexual, e raramente ocorre em indivíduos com menos de 60 anos de idade ${ }^{8}$, mas pode afetar crianças e adolescentes ${ }^{9}$. A incidência é de 21,7 casos/milhão ${ }^{10}$, e a taxa de mortalidade em um ano para pacientes com penfigóide bolhoso varia de $19 \%$ a $38 \%{ }^{4}$. Devido à faixa etária envolvida, à ocorrência concomitante de distúrbios neurodegenerativos, uso de doses elevadas de corticosteróides orais e propensão a malignidades e tromboembolismo venoso, a mortalidade por penfigóide bolhoso apresenta tendência crescente. Portanto, é de grande importância descobrir novas metas para reduzir sua morbidade e mortalidade ${ }^{6}$.

As manifestações clínicas incluem prurido e lesões urticadas e eritematosas que mais tarde se desenvolvem em bolhas subepidérmicas grandes e tensas ${ }^{11}$, frequentemente nas extremidades $^{5}$. Esta doença envolve principalmente a pele, mas ocasionalmente pode acometer olhos, boca e órgãos genitais; o penfigóide bolhoso clássico é clinicamente caracterizado por grandes bolhas (1-3 cm), tensas, serosas ou hemorrágicas que aparecem em lesões eritematosas, urticárias ou eczematosas ${ }^{12}$. A erupção do penfigóide bolhoso induzida por medicação é similar à forma idiopática, e pode ser desencadeado por medicações como furosemida, inibidores da enzima conversora da angiotensina, penicilina, ampicilina, cloroquina, psoralenos e sulfassalazina ${ }^{13}$.

O penfigoide bolhoso pode simular várias dermatoses e ser clinicamente indistinguível de outras doenças bolhosas, por isso, além da história médica e exame físico ${ }^{14}$ o diagnóstico de doenças bolhosas autoimunes é confirmado por estudos histológicos e imunopatológicos ${ }^{8}$. As lesões biopsiadas exibem bolhas subepidérmicas, com infiltração inflamatória densa de eosinófilos, basófilos, neutrófilos, linfócitos e mastócitos na derme, sendo a presença de eosinófilo sugestiva da doença ${ }^{6}$. Há também infiltrado linfocitário perivascular e intersticial com muitos eosinófilos e edema dérmico; espongiose discreta pode estar presente na periferia da bolha ${ }^{13}$. A imunofluorescência direta é o método mais sensível para 0 diagnóstico de penfigóide bolhoso, em que a lesão apresenta deposição linear de imunoglobulina $G$ (IgG), complemento C3 e IgE. A imunofluorescência indireta revela uma deposição linear de $\operatorname{lgG}^{6}$.

Atualmente, os corticosteróides tópicos ou sistêmicos, com ou sem agentes imunossupressores, ainda são os principais medicamentos para tratamento do penfigóide bolhoso $^{8,15}$. A terapia destina-se apenas a aliviar os sintomas e reduzir a duração da doença; a cicatrização ocorre após algumas semanas ou meses de tratamento ${ }^{14}$.

0 objetivo desse trabalho foi compreender os diagnósticos diferenciais do 
penfigóide bolhoso e sua etiologia como farmacodermia.

\section{RELATO DO CASO}

Paciente do sexo feminino, 85 anos, com antecedentes de etilismo, tabagismo, hipertensão arterial sistêmica e doença arterial obstrutiva periférica, com histórico de amputação distal de membro inferior direito há 3 meses, procurou o pronto atendimento de um hospital de Presidente Prudente referindo bolhas difusas pelo corpo há aproximadamente 2 meses, após internação prévia no serviço para amputação do membro. Foi medicada com corticoide (prednisona $5 \mathrm{mg}$ ) e antibiótico (cefalexina) via oral, sem melhora, e encaminhada ao ambulatório de Dermatologia para avaliação.

Ao exame dermatológico observou-se a presença de bolhas tensas íntegras e rotas, crostas e máculas hipocrômicas em pernas, antebraços e mãos (áreas fotoexpostas) (Figuras 1 e 2), sem queixa de dor ou prurido. Constava-se em prontuário uso recente, anterior ao aparecimento das bolhas, de levofloxacino, ciprofloxacino, clindamicina, cefalexina, devido à amputação realizada, além de cilostazol, clopidogrel, hidroclorotiazida, metildopa, sinvastatina e ácido acetilsalicílico (AAS) de longa data. Paciente foi então internada para melhor investigação da etiologia, sob hipóteses diagnósticas de Penfigóide Bolhoso, Pelagra, Porfiria Cutânea Tarda, Epidermólise Bolhosa Adquirida e Lúpus Eritematoso Cutâneo Bolhoso.

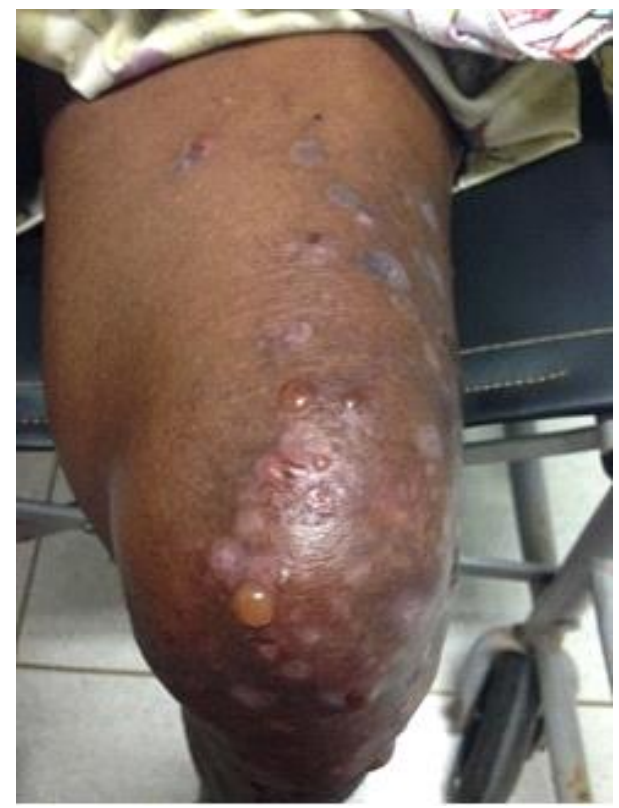

Figura 1. Numerosas bolhas íntegras e rotas e máculas hipocrômicas.

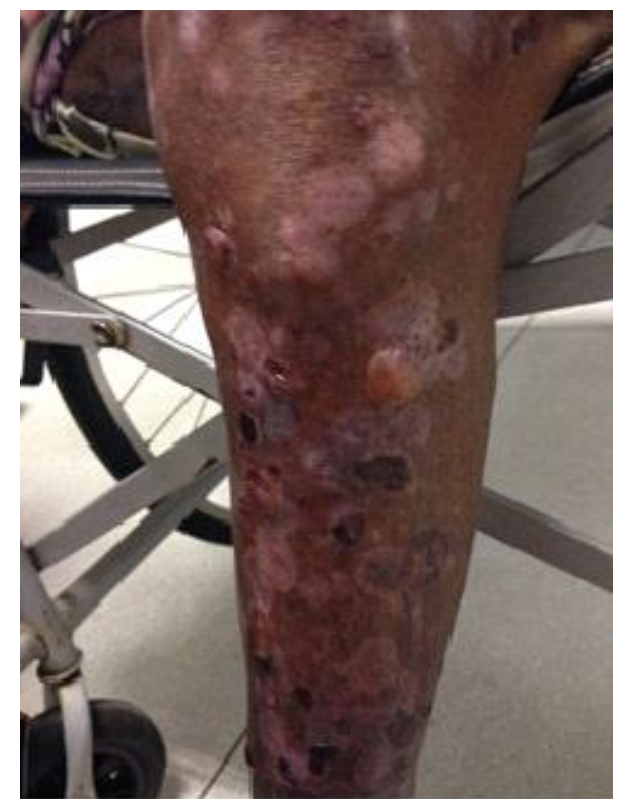

Figura 2. Presença de bolhas, crostas e máculas hipocrômicas em membro inferior.

O exame físico evidenciava sinais vitais normais e os exames laboratoriais iniciais demonstraram eosinofilia, e excluíram infecções em sítios comuns. Foram solicitadas dosagens de coproporfirina e uroporfirina, que se apresentaram negativos, descartando a hipótese de porfiria cutânea tarda, e foi realizada biópsia cutânea de uma das bolhas, cujo exame histopatológico revelou segmento de pele com espongiose moderada e formação de bolha na camada córnea com exocitose de alguns eosinófilos. Na derme, notou-se infiltrado inflamatório linfomononuclear leve perivascular com presença de eosinófilos e edema discreto, não havendo critérios morfológicos para porfiria cutânea tarda ou pelagra, sugerindo diagnóstico de penfigóide bolhoso de etiologia farmacológica.

O uso de antibióticos foi suspenso e mantidas as medicações de uso contínuo para tratamento de hipertensão arterial e doença arterial obstrutiva periférica. Durante a internação, paciente evoluiu estável e sem intercorrências, porém com surgimento de algumas novas bolhas, iniciando-se uso de prednisona (50 $\mathrm{mg} / \mathrm{dia}$, via oral), obtendo-se melhora das lesões com diminuição do número de bolhas após 2 dias de tratamento. Ao 50 dia de tratamento, a paciente apresentou notável melhora, restando poucas bolhas e máculas hiper e hipocrômicas. Assim, foi prescrita dexametasona tópica ( 2 vezes ao dia) e mantevese a prednisona.

A condição da pele da paciente apresentou melhoras significativas, evoluindo no 
sexto dia para ausência de bolhas, observando-se apenas crostas e máculas hiper/hipocrômicas residuais (Figura 3). Recebeu alta após 10 dias de internação mantendo-se a prednisona $(50 \mathrm{mg} / \mathrm{dia}$ via oral), com retorno ambulatorial em 3 semanas. Paciente retornou sem lesões e assintomática, sendo então iniciada redução gradual da dose de prednisona. Foi realizada a suspensão da medicação e, até o presente, não houve surgimento de novas lesões.

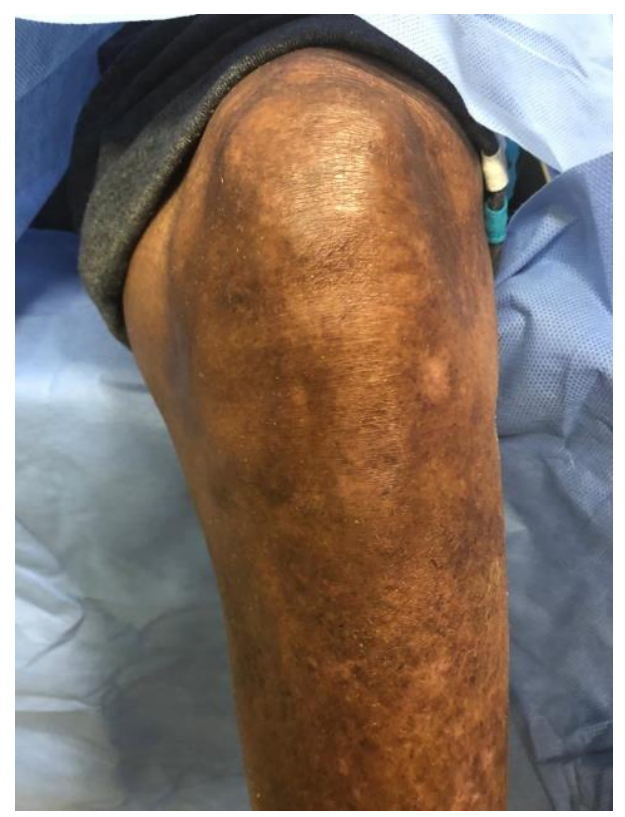

Figura 3. Após o tratamento, apresenta apenas máculas hiper e hipocrômicas residuais.

O relato foi avaliado e aprovado pelo Comitê de Ética em Pesquisa (CEP) da Universidade do Oeste Paulista - UNOESTE, sob o protoloco CAAE n. 91078918.6.0000.5515.

\section{DISCUSSÃO}

O quadro apresentado pela paciente sugeriu, inicialmente, diversas hipóteses diagnósticas, sendo estabelecido o diagnóstico de penfigóide bolhoso, com auxílio do exame histopatológico. A paciente relatada se enquadra no perfil epidemiológico mais comum para o aparecimento dessa dermatose bolhosa, conforme salientam alguns autores ${ }^{4-6,8}$, pois é mais frequente após os 70 anos de idade. Quanto às características dermatológicas, foram encontradas bolhas grandes e tensas sobre lesões eritematosas, conforme foi descrito por outros literatos $^{11,12}$ como lesões características do penfigóide bolhoso. Através da avaliação histológica, observou-se a formação de bolha na camada córnea com exocitose de alguns eosinófilos, e infiltrado inflamatório linfomononuclear com presença de eosinófilos, o que coincide com as características levantadas por estudiosos ${ }^{6,13}$. Além do mais, a eosinofilia encontrada no hemograma da paciente é uma característica presente em portadores de penfigóide bolhoso ${ }^{16}$.

Assim, após o resultado da biópsia e das dosagens de porfirina concluiu-se o diagnóstico de penfigóide bolhoso induzido por fármaco, caracterizando uma farmacodermia. No penfigóide bolhoso induzido por drogas, as características clínicas, histológica e imunopatológicas são similares àquelas do penfigóide bolhoso idiopático ${ }^{17}$.

A etiologia do penfigóide bolhoso ainda não é totalmente estabelecida, no entanto, é conhecido que algumas drogas são capazes de induzir o seu desenvolvimento ${ }^{5}$. Seu mecanismo pode ser explicado por duas situações: medicamentos contendo sulfidrila pode levar à divisão dermoepidérmica direta com ou sem anticorpos; e algumas drogas indutoras podem diminuir a atividade das células $T$ supressoras, estimulando a hiperprodução de auto-anticorpos contra os antígenos ${ }^{18}$. No entanto, uma predisposição genética deve ser suposta, visto que apenas algumas pessoas desenvolvem este tipo de lesões após o uso de tais drogas ${ }^{19}$.

0 penfigóide bolhoso induzido por fármaco já foi relacionado com vários medicamentos por diversos autores: associação do penfigóide bolhoso com drogas como fenacetina, penicilamina, furosemida, cloroquina, espironolactona, enalapril, losartana e hidroclorotiazida ${ }^{10}$; casos de penfigóide bolhoso induzidos por ciprofloxacino e levofloxacino ${ }^{19} \mathrm{e}$ associação com o uso de cefalexina ${ }^{17}$.

Foi estabelecido que, para um medicamento ser o gatilho para penfigóide bolhoso, deve ser: iniciado dentro de um ano do diagnóstico do penfigóide bolhoso, utilizado por mais de duas semanas e não interrompido por mais de um mês antes do diagnóstico. Estas condições basearam-se nos pressupostos de que medicamentos crônicos, exposições curtas e retirada antes do início da doença são improváveis de acionar o penfigóide bolhoso ${ }^{20}$. A paciente utilizou diversas medicações que podem ter elucidado com o aparecimento das lesões bolhosas. Dessas, encontrou-se na literatura relação com cefalexina, hidroclorotizaida, AAS, levofloxacino e ciprofloxacino, no entanto, pela 
análise das variáveis do caso pressupõem-se que as quinolonas foram responsáveis pelo desenvolvimento do quadro.

O tratamento da paciente foi iniciado com corticoide sistêmico, obtendo melhora do quadro. Foi adicionado corticoide tópico a este paciente, alcançando resposta satisfatória. Durante a internação a paciente não apresentou intercorrências e obteve resolução do quadro.

\section{CONCLUSÃO}

Foi relatado o caso de uma paciente de 85 anos que apresentou lesão bolhosa com características clínicas e histológicas de penfigóide bolhoso induzido por medicação. Há relatos na literatura de penfigóide bolhoso provocado por diversas medicações utilizadas pela paciente, como cefalexina, hidroclorotiazida, AAS, ciprofloxacino e levofloxacino; pela relação do uso da medicação com o aparecimento da reação, podemos concluir que as quinolonas foram responsáveis pelo quadro da paciente, no entanto, não foi possível afirmar sobre a droga específica, sendo uma oportunidade para o desenvolviemnto de estudos futuros.

As reações cutâneas estão entre as mais frequentes reações adversas relacionadas a drogas, como foi relatado no presente trabalho; assim, ressalta-se a importância do reconhecimento das reações medicamentosas existentes e de sua apresentação mais provável na pele, além do questionamento, na anamnese, do uso de medicamentos, garantindo assim diagnóstico precoce seguido da suspensão da medicação, porque a retirada do agente causador está relacionada à melhora do quadro.

\section{REFERÊNCIAS}

1. Antonio RJ, Antonio RC. Farmacodermias. Dermatologia Ibero-Americano Online, São Paulo, 2010. Acesso em: ago 2018. Disponível em: http://piel-I.org/libreria/item/921

2. Silva SM, Roselino AMF. Reações de hipersensibilidade a drogas (farmacodermia). Rev. Med. 2003;36(2):460-71.

3. Anastasopoulou A, Papaxoinis G, Diamantopoulos $P$, Christofidou E, Benopoulou O, Stratigos A, Gogas H. Bullous Pemphigoid-like skin lesions and overt eosinophilia in a patient with melanoma treated with nivolumab: case report and review of the literature. J Immunother. 2018;41(3):164-7. DOI:
http://dx.doi.org/10.1097/CJI.000000000000021 $\underline{0}$

4. Atzmony L, Mimouni I, Reiter O, Leshem YA, Taha O, Gdalevich M, Hodak E, Mimouni D. Association of bullous pemphigoid with malignancy: A systematic review and metaanalysis. J Am Acad Dermatol. 2017;77(4):691-9. DOI:

\section{http://dx.doi.org/10.1016/i.jaad.2017.05.006}

5. Fang $H$, Shen $S$, Zheng $X$, Dang $E$, Zhang J, Shao $S$, Qiao $P$, Li $Q$, Wang $H$, Li C, Sun L, Wang G. Association of HLA class I and class II alleles with bullous pemphigoid in Chinese Hans. J Dermatol Sci. 2018;89(3):258-62. DOI: http://dx.doi.org/10.1016/j.jdermsci.2017.11.014

6. Liu Y, Li L, Xia Y. BP180 is critical in the autoimmunity of bullous pemphigoid. Front Immunol. 2017;8:1752. DOI: http://dx.doi.org/10.3389/fimmu.2017.01752

7. Hammers CM, Stanley JR. Mechanisms of disease: pemphigus and bullous pemphigoid. Annu Rev Pathol. 2016;11:175-97. DOI: http://dx.doi.org/10.1146/annurev-pathol$\underline{012615-044313}$

8. Ding Shu, Deng Qiancheng, Xiang Yaping, Chen Jing, Huang Jinhua, Lu Jianyun. Bullous pemphigoid associated with milia, increased serum IgE, autoantibodies against desmogleins, and refractory treatment in a young patient. An Bras Dermatol. 2017;92(5 Suppl 1):34-6.

9. Zhao CY, Murrell DF. Blistering diseases in neonates. Curr Opin Pediatr. 2016;28(4):500-6. DOI:

http://dx.doi.org/10.1097/MOP.0000000000000 $\underline{381}$

10. Warner $\mathrm{CB}$, Kwak $\mathrm{Y}$, Glover $\mathrm{MH}$, Davis LS. Bullous pemphigoid induced by hydrochlorothiazide therapy. J Drugs Dermatol. 2014;13(3):360-2 .

11. Kwan Z, Lai YN, Ch'ng CC, Tan AH, Tan LL, Robinson $S$, Rokiah I. The association between bullous pemphigoid and neurological disorders in a selected malaysian population. Med J Malaysia. 2015;70(2):81-5.

12. Schmidt E, Zillikens D. Pemphigoid diseases. 
Lancet. 2013;381(9863):320-32. DOI: http://dx.doi.org/10.1016/S0140-6736(12)61140$\underline{4}$

13. Rossi G. Aspectos clínicos e dermatoscópicos das farmacodermias. [Dissertação]. Mestrado no Programa de Pós-Graduação em Medicina: Ciências Médicas. Faculdade de Medicina da Universidade Federal do Rio Grande do Sul, Porto Alegre, RS, 2017.

14. Reisfilho EGM, Silva TA, Aguirre LHL, Reis CMS. Bullous pemphigoid in a 3-month-old infant: case report and literature review of this dermatosis in childhood. An Bras Dermatol. 2013;88(6):961-5.

15. Bagci IS, Horváth ON, Ruzicka T, Sárdy M. Bullous pemphigoid. Autoimmun Rev. 2017;16(5):445-55. DOI: http://dx.doi.org/10.1016/i.autrev.2017.03.010

16. Geiss Steiner J, Trüeb RM, Kerl K, Mühleisen B, French LE, Hofbauer GF. Ecthymagangrenosum-like bullous pemphigoid. Dermatology. 2010;221(2):142-8. DOI: http://dx.doi.org/10.1159/000316098

17. Czechowicz RT, Reid CM, Warren LJ, Weightman W, Whitehead FJ. Bullous pemphigoid induced by cephalexin Australasian. Australas J Dermatol. 2001;42(2):132-5.

18. Ma HJ, Hu R, Jia CY, Yang Y, Song LJ. Case of drug-induced bullous pemphigoid by levofloxacino. J Dermatol. 2012;39(12):1086-7. DOI: $\quad$ http://dx.doi.org/10.1111/j.13468138.2012.01636.x

19. Cozzani E, Chinazzo C, Burlando M, Romagnoli $M$, Parodi A. Ciprofloxacin as a trigger for bullous pemphigoid: the second case in the literature. Am J Ther. 2016;23(5):e1202-4. DOI: http://dx.doi.org/10.1097/MJT.00000000000002 $\underline{83}$

20. Tan CW, Pang $Y$, Sim B, Thirumoorthy $T$, Pang $\mathrm{SM}$, Lee HY. The association between drugs and bullous pemphigoid. $\mathrm{Br} J$ Dermatol. 2017;176(2):549-51. DOI: http://dx.doi.org/10.1111/bjd.15195 